\title{
Shaping colloidal bananas to reveal biaxial, splay-bend nematic and smectic phases
}

\author{
Carla Fernández-Rico ${ }^{1}$, Massimiliano Chiappini ${ }^{2}$, Taiki Yanagishima ${ }^{1}$, \\ Heidi de Sousa ${ }^{1}$, Dirk G.A.L. Aarts ${ }^{1}$, Marjolein Dijkstra ${ }^{2}$ and Roel P.A. Dullens ${ }^{1 *}$ \\ ${ }^{1}$ Department of Chemistry, Physical and Theoretical Chemistry Laboratory, University of Oxford, \\ South Parks Road, Oxford OX1 3QZ, United Kingdom \\ ${ }^{2}$ Soft Condensed Matter, Debye Institute for Nanomaterials Science, Department of Physics, \\ Utrecht University, Princetonplein 1, 3584 CC Utrecht, The Netherlands \\ *E-mail: roel.dullens@chem.ox.ac.uk
}

July 1, 2020

Understanding the impact of curvature on the self-assembly of elongated microscopic building blocks, such as molecules and proteins, is key to engineering functional materials with pre-designed structure. We develop model 'bananashaped' colloidal particles with tuneable dimensions and curvature, whose structure and dynamics are accessible at the particle level. By heating initially straight SU-8 rods, we induce a controllable shape deformation that causes the rods to buckle into banana-shaped particles. We elucidate the phase behavior of differently curved colloidal bananas using confocal microscopy. While highly curved bananas only form isotropic phases, less curved bananas exhibit very rich phase behavior including biaxial nematic, polar and antipolar smectic-like phases, and even the long predicted, elusive splay-bend nematic phase. 
Curvature has an enormous impact on the functionality and self-assembly of elongated microscopic building blocks $(1,2)$. In the biological world, for instance, curved rod-shaped bacteria outperform their straight counterparts in surface-colonisation and swimming efficiency, which makes them ubiquitous in marine environments $(3,4)$. Many cellular functions, such as cell division or endocytosis, rely on the ability of 'banana-shaped' proteins to generate curvature in cell membranes $(5,6)$. Curvature is also of key importance at the molecular scale where, for example, banana-shaped or bent-core molecules exhibit a fascinating range of new liquid crystalline phases with distinctive features such as supramolecular chirality and polarity (7-9). This has led to a surge of interest in banana-shaped liquid crystals from both a fundamental and technological point of view. They are not only ideal systems to study, for example, the spontaneous chiral symmetry breaking in systems of achiral molecules (10-13), but also excellent candidates for achieving faster switching speeds in display technologies $(14,15)$.

Over the past two decades, more than fifty new banana-shaped liquid crystalline phases have been reported depending primarily on the molecular curvature (7-9). The vast majority of these are smectic ( $S m$ ) phases, as the curved shape of the constituting molecules promotes their locking into smectic layers $(8,9)$. The large stability of $S m$ phases has also been observed in experiments of colloidal 'boomerang-like' particles (16), and in computer simulations of similarly shaped particles (17). The rather uncommon observation of nematic phases in bananashaped systems $(18,19)$ has been of interest for the past 20 years, not least because of their potential to form chiral and biaxial nematic phases $(13,17,20,21)$. Examples of chiral and biaxial nematic phases are the twist-bend $\left(N_{T B}\right)$ and the splay-bend $\left(N_{S B}\right)$ nematic phases, respectively, in both of which the particle orientation is modulated in space. While in the $N_{T B}$ phase the particles exhibit a periodic twist in space resulting in a chiral phase, the $N_{S B}$ phase exhibits periodic splay and bend modulations of the particle orientation in a single plane, therefore showing biaxiality but not chirality (12). 
The $N_{T B}$ and $N_{S B}$ phases were postulated over forty years ago by Meyer (22), and later independently by Dozov (12), who suggested that bend deformations in the orientation field of banana-shaped particles should be accompanied either by twist or splay deformations to fill 3D space. While the $N_{T B}$ phase has been observed in thermotropic liquid crystals (see e.g. (23-25)) the existence of the $N_{S B}$ phase is still to be confirmed experimentally. Computer simulations suggested that the $N_{S B}$ phase can be found in systems of hard boomerangs, i.e. rod-like particles with a sharp kink, if the $S m$ phase is destabilised by polydispersity in the particle length or by smooth curvature in the particle shape (17). Experimentally studying the impact of features such as the curvature on the structure of banana-shaped systems at the particle level is thus crucial for a deeper understanding of the formation and properties of these phases. However, banana-shaped liquid crystals are typically characterised using birefringence and Xray diffraction techniques, where direct information about structural details is not available at the molecular scale. This prompts the need for a colloidal analogue, where such microscopic structural information is readily accessible using optical microscopy. However, despite the abundance of liquid crystal forming colloidal particles such as rods, boomerangs and platelets (26-28), there is no system of smoothly curved colloidal rods available. As such, the rich phase behavior predicted for banana-shaped particles is still to be experimentally uncovered at the particle level.

In this work, we develop a method to produce, in bulk, fluorescent curved colloidal rods - colloidal bananas - with tuneable dimensions and curvature. Our method relies on the temperature-driven buckling of initially straight SU-8 rods into colloidal bananas. The extent of the shape deformation, and hence the final curvature of the particles, is controlled by the rigidity of the SU-8 rods during the heating. Using confocal microscopy, we elucidate the phase behavior of three differently curved banana-shaped particles at the single particle level. While isotropic phases are found for highly curved bananas, very rich phase behavior, including 
biaxial nematic, splay-bend nematic and polar and antipolar smectic-like structures, is observed for less curved bananas.

\section{Shaping colloidal SU-8 particles: from rods to banana-shaped particles}

Our method to produce colloidal SU-8 banana-shaped particles consists of four main steps, which are illustrated in Fig. 1A: (I) synthesis of rods, (II) UV-exposure, (III) heating and (IV) UV-curing. In the first step, polydisperse straight SU-8 rods ( $L \sim 20 \mu m$, see Fig. 1B) are synthesised by vigorously shearing an emulsion of SU-8 droplets in a viscous medium $(29,30)$. During the second step, the resulting rods are partially cross-linked via the ring-opening reaction of the epoxy groups under low-intensity UV-light (See Sec.2 SM). The degree of cross-linking in the rods is controlled by the UV-exposure time, $t_{\mathrm{UV}}$, as confirmed by the IR spectra shown in Fig. S3, where the peak corresponding to the epoxy groups decreases with increasing exposure time. In the third step, the deformation into banana-shaped particles is induced by heating the rods in a $95^{\circ} \mathrm{C}$ oven, resulting in smoothly curved polydisperse colloidal bananas (see Fig. 1C and $\left.\mathrm{D}, t_{\mathrm{UV}}=45 \mathrm{~min}\right)$. Finally, the particles are fully cross-linked via high intensity UV-light exposure, yielding highly stable particles that can be dispersed in both aqueous and non-aqueous solvents.

The formation of banana-shaped particles is controlled by the interplay between the crosslinking density of the SU-8 rods, tuned by the UV-exposure time, and the interfacial forces induced during the heating step. The cross-linking density of SU-8 dictates its rigidity and glass transition temperature, and both increase with longer UV-exposures $(31,32)$. In our experiments, we observe three distinct responses upon heating, depending on the UV-exposure time. At short exposure times $\left(t_{\mathrm{UV}}=1 \mathrm{~min}\right.$, Fig. 2A), i.e. low cross-linking densities, the heating of the rods results in the rounding of their sharp edges (see Fig. 2C), suggesting that the rods are heated to a temperature above their glass transition temperature. The interfacial forces as- 
sociated with the hemispherical particle ends drive the collapse of the rods into spheres, rather than bananas, in order to minimise their surface energy (33-35). This collapse takes place via a sphero-cylindrical intermediate state, as shown by the snapshots taken during heating in Fig. 2A (see also movie S1). From the corresponding scanning electron microscopy (SEM) images, shown in Fig. 2C, we find that the particle length decreases, and the diameter increases (see Fig. 2F), but that the particle volume remains constant during heating (see Fig. S5). At intermediate exposure times $\left(t_{\mathrm{UV}}=25 \mathrm{~min}\right.$, Fig. $\left.2 \mathrm{~B}\right)$, the heating of the rods still results in the formation of round edges (see Fig. 2D), which leads to an initial decrease of the rod length and an increase of the rod diameter (Fig. 2F). However, the increased cross-linking density of the rods, i.e. the higher rigidity, leads to the formation of banana-shaped particles via buckling, as directly visualised during the heating process using confocal microscopy (see Fig. 2B, and movie S2). The buckling manifests itself by the appearance of curvature after heating for $20 \mathrm{~min}$, where the particle length and diameter no longer change significantly (see Fig. 2F). At large exposure times $\left(t_{\mathrm{UV}}=120 \mathrm{~min}\right.$, Fig. $\left.2 \mathrm{E}\right)$, i.e. high cross-linking densities, no rounding of the edges is observed, suggesting that the rods are heated to temperatures below their glass transition temperature. Consequently, no shape deformation is observed and the length, diameter and curvature are all constant during the heating (see Fig. 2F). Finally, carrying out the experiments in the dark, i.e. no cross-linking takes place, always results in the formation of spheres (see Fig. S4), which thus confirms the crucial role of the interplay between interfacial forces and the differing UV-induced rigidity of the rods on controlling the final particle shape.

\section{Morphological state diagram}

The ability to systematically control the final particle shape using both UV-exposure and heating time (see Sec.1 SM) is summarised in the morphological state diagram shown in Fig. 3A. Within the banana regime, we show that both the dimensions and curvature of the resulting banana- 
shaped particles can be controlled by tuning the UV-exposure time. This is observed in the SEM images presented in Fig. 3C-E, where the bananas obtained using UV-exposure times of $t_{\mathrm{UV}}=15,25$ and $45 \mathrm{~min}$, respectively, are shown for a constant heating time of $30 \mathrm{~min}$. We find that with longer UV-exposure times, the particle length $L$ (see inset Fig. 3D), increases from $8 \mu \mathrm{m}$ to $14 \mu \mathrm{m}$, while the diameter and curvature decrease from $1 \mu \mathrm{m}$ to $0.7 \mu \mathrm{m}$ and from $0.25 \mu \mathrm{m}^{-1}$ to $0.07 \mu \mathrm{m}^{-1}$, respectively (see Fig. 3B and Fig. S6). This corroborates the fact that the rigidity of the rods increases and thus the extent of buckling decreases with UV-exposure time. Inherent to the polydisperse nature of the initial SU-8 rods $(29,30)$, the resulting colloidal bananas are also polydisperse in length, diameter and curvature, with typical polydispersities of $30 \%, 20 \%$ and $30 \%$, respectively (see Table S1).

\section{Phase behavior of colloidal bananas}

We study the phase behavior of banana-shaped particles, and how this is affected by curvature, by preparing concentrated samples of three differently-curved colloidal bananas and imaging them using confocal microscopy. Whilst the three-dimensional samples are typically $\sim 50 \mu \mathrm{m}$ thick, we image the system relatively close to the bottom wall of the sample container to which the bananas align, as this facilitates a quantitative two-dimensional structural analysis at the particle level (see SM Sec. 4.2 for details). In Fig. 4, we present confocal microscopy images of the structures formed by the three differently curved colloidal bananas at different packing fractions $\phi$. The most curved bananas $\left(\kappa=0.25 \mu \mathrm{m}^{-1}\right)$ only display isotropic phases $(I)$ at all packing fractions, as shown in Fig. 4A (see Fig. S9 for confocal images of the system at all packing fractions studied). Although small smectic domains are observed at high packing fractions, the combination of polydispersity and small opening angles of these bananas (see Table S1) suppresses the formation of any ordering at larger length scales. This is corroborated by the flat distribution of polar orientation $\theta$ (see inset in Fig. $4 \mathrm{~A}_{5}$ ) of the bananas, $p(\theta)$, in 
Fig. $4 \mathrm{~A}_{5}$, and the lack of orientational correlations over space in the overlays in Fig. $4 \mathrm{~A}_{1}$ $\mathrm{A}_{4}$, where the bananas are coloured according to their polar orientation. Note that due to the alignment of the bananas to the wall, the orientation of the long axis is directly related to the polar orientation via a $\pi / 2$ rotation (see Fig. S7).

The bananas with intermediate curvature, $\kappa=0.10 \mu \mathrm{m}^{-1}$, also exhibit isotropic ordering at low packing fractions (Fig. $4 \mathrm{~B}_{1}$ ), but self-assemble into biaxial nematic and polar and antipolar smectic-like phases at higher packing fractions as shown in Fig. $4 \mathrm{~B}_{2}-\mathrm{B}_{4}$ (see Fig. S12 for images at all packing fractions). The onset of orientational ordering in the nematic and smectic phases manifests itself as the emergence of peaks in $p(\theta)$ above $\phi=0.63$, as shown in Fig. $4 \mathrm{~B}_{5}$. The biaxial nature of this ordering is inherent to the orthogonality of the long and polar axes of the bananas due to their alignment to the wall (see Fig. S7). In particular, the biaxial nematic phase $\left(N_{b}\right)$ observed at $\phi=0.63$ (Fig. $\left.4 \mathrm{~B}_{2}\right)$ is characterized by two peaks in $p(\theta)$, which correspond to the two polar orientations the bananas show in this phase. At higher packing fractions, $\phi=0.79$ (Fig. 4B ${ }_{3}$ ), the emergence of smectic domains, with some local polar smectic ordering, is observed (36). The polydomain structure at this packing fraction is likely due to kinetic effects (see SM Sec. 4.2) and results in a single broad peak in $p(\theta)$. Antipolar smectic ordering is observed at $\phi=0.82\left(\mathrm{Fig} .4 \mathrm{~B}_{4}\right)$, which leads to a double peak in $p(\theta)$ due to the neighbouring layers exhibiting alternating polar orientation, as is also evident from the overlay in Fig. $4 \mathrm{~B}_{4}$.

Even richer phase behavior is observed for the least curved bananas $\left(\kappa=0.07 \mu \mathrm{m}^{-1}\right)$, as shown in Fig. 4C (see Fig. S15 for images at all packing fractions). As expected, the isotropic phase is observed at low packing fractions. Upon increasing packing fraction, the system starts to exhibit biaxial nematic ordering (Fig. $4 \mathrm{C}_{2}$ and $4 \mathrm{C}_{3}$ ), as is evident from the development of peaks in $p(\theta)$ (Fig. $4 \mathrm{C}_{5}$ ), and eventually forms layered antipolar biaxial smectic structures at $\phi=0.79$ (Fig. $4 \mathrm{C}_{4}$ ). We also observe different types of defects as shown in Fig. S24. Most interestingly, however, the biaxial nematic phase of bananas with this curvature (Fig. $4 \mathrm{C}_{3}$ ) 
exhibits a strong spatial modulation of the particle orientations, which is reminiscent of the elusive biaxial splay-bend nematic phase.

\section{Splay-bend nematic phase in colloidal bananas}

To distinguish the splay-bend nematic phase from the biaxial nematic and smectic phases, we measure the spatial modulation of both the particle positions and orientations along the nematic director $\hat{\mathbf{n}}$, defined as the average particle orientation of the phase (see SM Sec. 7). To this end, we determine the center of mass positions and the particle orientations $\hat{\mathbf{u}}_{\mathbf{i}}$ (see inset Fig. $5 \mathrm{~A}_{1}$ ) of all the particles as presented in Fig. $5 \mathrm{~A}_{1}$ and $5 \mathrm{~A}_{2}$, respectively, for the least curved bananas at $\phi=0.67$. From these, we extract the number density profile and the nematic director field along the nematic director $\hat{\mathbf{n}}$ (see SM Sec. 7), which are shown as the blue line and the white arrows in Fig. $5 \mathrm{~A}_{3}$, respectively. Note that the $y$-axis is defined to be parallel to $\hat{\mathbf{n}}$. In Fig. 5B we present these measurements for the five highest packing fractions $(\phi=0.60-0.79)$ for the bananas with the lowest curvature $\left(\kappa=0.07 \mu \mathrm{m}^{-1}\right)$. Firstly, we observe that the number density profiles, $\rho(y)$, are mostly flat, characteristic for nematic phases, except for the highest packing fraction $(\phi=0.79)$, where clear peaks develop due to the organisation of the bananas into positionally ordered smectic layers. Consistently, the global smectic order parameter (see SM Sec. 7) only increases at $\phi=0.79$, as shown in Fig. 5E, confirming the smectic nature of this phase. Secondly, we observe a clear modulation of the nematic director field, $\hat{\mathbf{n}}(y)$, for all five packing fractions as is evident from the periodic fluctuations in the orientations of the white arrows in Fig. 5B.

A detailed characterisation of the measured $\hat{\mathbf{n}}(y)$ is shown in Fig. $5 \mathrm{C}$, where the spatial modulations of its components parallel $\left(n_{y}\right)$ and perpendicular $\left(n_{x}\right)$ to the nematic director are shown for all five packing fractions. To unambiguously identify the splay-bend nature of these phases, we fit the measured nematic director field with the theoretical expression for the director field of 
a splay-bend nematic phase (12), given by $\hat{\mathbf{n}}(y)=\left\{\sin \left(\theta_{0} \sin \left(\frac{2 \pi}{p} y\right)\right), \cos \left(\theta_{0} \sin \left(\frac{2 \pi}{p} y\right)\right)\right\}$, where $\theta_{0}$ and $p$ are the amplitude and pitch-length of the modulation, respectively (12) (see Fig. S18).

As shown in Fig. 5C, we find that our experimental data for $n_{x}$ and $n_{y}$ are remarkably well described by the expression for $\hat{\mathbf{n}}(y)$ for all packing fractions in the range $\phi=0.60-0.79$, confirming the splay-bend nature of the director field. However, for $\phi<0.67$, the amplitude $\theta_{0}$ and pitch-length $p$, obtained from these fits, increase with increasing $\phi$ (see Fig. 5D), in contrast to what is expected for the splay-bend nematic phase (17). Only for $\phi \geq 0.67, \theta_{0}$ and $p$ are directly proportional to each other and decrease with increasing $\phi$ (see Fig. 5D), in agreement with computer simulations of the splay-bend nematic phase (17), suggesting that the splay-bend nematic phase forms at $\phi \geq 0.67$. This is corroborated by the decrease in the global nematic order parameter (see SM Sec.7) at $\phi=0.67$, consistent with the onset of significant splaybend modulations of the particle orientations, resulting in a decrease of the global alignment of particles (see Fig. 5E). For our least curved colloidal bananas $\left(\kappa=0.07 \mu \mathrm{m}^{-1}\right)$ it is thus clear that they undergo a transition from the biaxial nematic phase to the modulated splay-bend nematic phase at $\phi \approx 0.67$, before going into the smectic phase at $\phi \simeq 0.79$. As far as we are aware, this is the first experimental observation of the splay-bend nematic phase (see also Fig. S23), which was predicted in 1976 (22). Finally, we demonstrate the 3D nature of the splaybend nematic phase in Fig. 5F, where a 3D confocal microscopy image shows the splay-bend deformations up to fifteen particle diameters into the bulk of our sample (see also Fig. S26).

The following picture of the phase behavior for our differently curved colloidal bananas emerges. For the most curved bananas $\left(\kappa=0.25 \mu \mathrm{m}^{-1}\right)$ only the isotropic phase is observed, for the bananas with intermediate curvature $\left(\kappa=0.10 \mu \mathrm{m}^{-1}\right)$ a phase sequence of $I-N_{b}-S m$ is found and the least curved bananas $\left(\kappa=0.07 \mu \mathrm{m}^{-1}\right)$ exhibit a $I-N_{b}-N_{S B}-S m$ phase sequence as a function of the packing fraction (see Fig. S27). The experimental observation of the splay- 
bend nematic phase confirms the importance of a smooth particle curvature or polydispersity for the stability of this phase (17), and also suggests that the typical sharp kink and purity of bentcore molecules could be one of the reasons as to why this phase has not yet been observed in molecular systems. While our results for the least curved bananas are largely consistent with the phase behavior found in computer simulations of similar banana-shaped particles (17), we do not observe the twist-bend nematic phase, which is predicted to occur between the nematic and splay-bend nematic phases (17). We attribute the absence of the $N_{T B}$ phase in the experimental phase behavior to a combination of the effect of gravity and the presence of a flat bottom wall in our sample cell. An external field like a gravitational or electric field may transform the $N_{T B}$ phase into a $N_{S B}$ phase in the case the nematic director is perpendicular to the external field (25). In addition, the presence of a flat wall favors biaxial order, and hence the $N_{S B}$ rather than the $N_{T B}$ phase may form close to the wall. The direct observation of the colloidal analogue of the $N_{T B}$ phase therefore still remains an exciting experimental challenge.

\section{References}

1. S. C. Glotzer, M. J. Solomon, Nature Materials 6, 557 (2007).

2. V. N. Manoharan, Science 349, 1253751 (2015).

3. A. Persat, H. A. Stone, Z. Gitai, Nature Communications 5, 3824 (2014).

4. R. Schuech, T. Hoehfurtner, D. J. Smith, S. Humphries, Proceedings of the National Academy of Sciences of the United States of America 116, 14440 (2019).

5. B. Qualmann, D. Koch, M. M. Kessels, EMBO Journal 30, 3501 (2011).

6. Y.-B. Shen, Z. Shen, Trends in Cell Biology 25, 59 (2015).

7. H. Takezoe, Y. Takanishi, Japanese Journal of Applied Physics 45, 597 (2006). 
8. R. A. Reddy, C. Tschierske, Journal of Materials Chemistry 16, 907 (2006).

9. A. Jákli, O. D. Lavrentovich, J. V. Selinger, Reviews of Modern Physics 90, 45004 (2018).

10. D. R. Link, et al., Science 278, 1924 (1997).

11. T. Sekine, et al., Journal of Materials Chemistry 7, 1307 (1997).

12. I. Dozov, Europhysics Letters 56, 247 (2001).

13. C. Greco, A. Ferrarini, Physical Review Letters 115, 147801 (2015).

14. N. A. Clark, S. T. Lagerwall, Applied Physics Letters 36, 899 (1980).

15. F. T. Niori. T, Sekine. T, Watanabe.J, T. H, J. Mater. Chem. 6, 1231 (1996).

16. Y. Yang, et al., Science Advances 4, 3aas8829 (2018).

17. M. Chiappini, T. Drwenski, R. van Roij, M. Dijkstra, Physical Review Letters 123, 068001 (2019).

18. M. Mathews, S. Kang, S. Kumar, Q. Li, Liquid Crystals 38, 31 (2011).

19. A. Jákli, Liquid Crystals Reviews 1, 65 (2013).

20. L. A. Madsen, T. J. Dingemans, M. Nakata, E. T. Samulski, Physical Review Letters 92, 145505 (2004).

21. C. Tschierske, D. J. Photinos, Journal of Materials Chemistry 20, 4263 (2010).

22. R. B. Meyer, in Molecular Fluids edited by R. Balian and G. Weil, pp. 271-343 (1976).

23. K. Adlem, et al., Physical Review E 88, 022503 (2013).

24. D. Chen, et al., Physical Review E 89, 022506 (2014). 
25. G. Pająk, L. Longa, A. Chrzanowska, Proceedings of the National Academy of Sciences of the United States of America 115, E10303 (2018).

26. F. M. Van Der Kooij, H. N. Lekkerkerker, Nature 406, 868 (2000).

27. Y. Yang, et al., Journal of the American Chemical Society 138, 68 (2016).

28. C. Fernández-Rico, T. Yanagishima, A. Curran, D. G. A. L. Aarts, R. P. A. Dullens, Advanced Materials 31, 1807514 (2019).

29. R. G. Alargova, K. H. Bhatt, V. N. Paunov, O. D. Velev, Advanced Materials 16, 1653 (2004).

30. R. G. Alargova, V. N. Paunov, O. D. Velev, Langmuir 22, 765 (2006).

31. F. Ru, J. F. Richard, Journal of Micromechanics and Microengineering 13, 80 (2003).

32. K. Ingolf Schiffmann, B. Carsten, International Journal of Materials Research 98, 397 (2007).

33. M. Caggioni, J. Lenis, A. V. Bayles, E. M. Furst, P. T. Spicer, Langmuir 31, 8558 (2015).

34. R. W. Style, A. Jagota, C.-y. Hui, E. R. Dufresne, Annual Review of Fluid Mechanics 8, 99 (2017).

35. J. Bico, E. Reyssat, B. Roman, Annual Review of Fluid Mechanics 50, 629 (2018).

36. Y. Lansac, P. K. Maiti, N. A. Clark, M. A. Glaser, Physical Review E 67, 011703 (2003).

37. I. D. Morrison, Colloids and Surfaces A 71, 1-37 (1993).

38. Y. Liu, et al., Langmuir 35, $7962-7969$ (2019). 
39. S. K. Sainis, V. Germain, C. O. Mejean and E. R. Dufresne, Langmuir 24, 1160-1164 (2007).

\section{Acknowledgments}

We thank Adam Edwards Stones and Louis Cortes for assistance with the image analysis. Funding: The ERC (ERC Consolidator Grant No. 724834 - OMCIDC) is acknowledged for financial support. M.C. and M.D. acknowledge financial support from the EU H2020-MSCA-ITN2015 project MULTIMAT (Marie Sklodowska-Curie Innovative Training Networks) (Project No. 676045). Author contributions: C.F.R. and R.P.A.D. conceived the project and designed the experiments. D.G.A.L.A. contributed to initiating the project and provided general expertise. C.F.R. and H.S. synthesized the colloidal bananas and T.Y. contributed to the interpretation of the synthesis mechanism. C.F.R. performed the microscopy experiments. C.F.R., M.C., M.D. and R.P.A.D. initiated the phase behaviour study. C.F.R. and M.C. wrote the image analysis routines and analysed the phase behaviour data, C.F.R., M.C., T.Y., M.D. and R.P.A.D. interpreted the data. M.D. and R.P.A.D. supervised the analysis of the phase behaviour. C.F.R. and R.P.A.D. wrote the manuscript and all co-authors commented on the manuscript. R.P.A.D. supervised the project. Competing interests: The authors declare no competing interests. Data and materials availability: All data are available in the main text or the supplementary materials.

\section{Supplementary materials}

Materials and Methods

Figs. S1 to S27

Table S1

References $(37-39)$

Movies S1 to S4 
Fig. 1. Synthesis of colloidal SU-8 banana-shaped particles. (A) Schematic showing the synthesis of SU-8 banana-shaped particles. In the first step, straight SU-8 rods are synthesised by shearing an emulsion of SU-8 droplets $(29,30)$. In the second step, the rods are partially cross-linked via exposure to UV-light. In the third step, the partially cross-linked rods are heated to induce a shape deformation into banana-shaped particles, before they are UV-cured in the fourth step. Scanning electron microscopy images of (B) the rods obtained after the synthesis with no heating and $(\mathrm{C})$ the colloidal SU-8 bananas $\left(t_{\mathrm{UV}}=45 \mathrm{~min}\right)$ obtained with heating. (D) Confocal microscopy image of the fluorescent colloidal SU-8 bananas. Scale bars are $10 \mu \mathrm{m}$.

Fig. 2. Particle shape deformations during heating. (A) Bright field and (B) confocal microscopy snapshots of the deformation of an SU-8 rod during heating at $95^{\circ} \mathrm{C}$ using a heating stage, for $(\mathrm{A}) t_{\mathrm{UV}}=1 \mathrm{~min}$ and $(\mathrm{B}) t_{\mathrm{UV}}=25 \mathrm{~min}$. Representative SEM images of the shape evolution of the SU-8 polymer rods during heating in a $95^{\circ} \mathrm{C}$ oven for $(\mathrm{C}) t_{\mathrm{UV}}=1 \mathrm{~min}$, (D) $t_{\mathrm{UV}}=25 \mathrm{~min}$ and (E) $t_{\mathrm{UV}}=120 \mathrm{~min}$. (F) Evolution of the dimensions and curvature of the originally straight SU-8 polymer rods during heating in a $95^{\circ} \mathrm{C}$ oven for $t_{\mathrm{UV}}=1 \mathrm{~min}$ (circles), $t_{\mathrm{UV}}=25 \mathrm{~min}$ (triangles) and $t_{\mathrm{UV}}=120 \mathrm{~min}$ (squares). Error bars represent the standard deviation of the mean value. Scale bars are (A-B) $10 \mu \mathrm{m}$ and (C-E) $5 \mu \mathrm{m}$.

Fig. 3. Tuning particle shape and controlling the curvature and dimensions of bananashaped particles. (A) Morphological state diagram of the SU-8 polymer particles as a function of UV-exposure and heating time with a heating temperature of $95^{\circ} \mathrm{C}$, where $S, B, R$ and $S c$ denote the sphere, banana, rod and sphero-cylinder regimes, respectively. (B) Length, diameter and curvature of the SU-8 particles after heating for $30 \mathrm{~min}$, see dashed line in (A), in a $95^{\circ} \mathrm{C}$ oven for $t_{\mathrm{UV}}=5,15,25,45,90$ and $120 \mathrm{~min}$. Error bars represent the standard deviation of the mean value and the different colors of the symbols correspond to the different regimes in (A). 
(C-E) SEM images of banana-shaped particles with decreasing curvature obtained when $t_{\mathrm{UV}}=$ 15, 25 and $45 \mathrm{~min}$. Scale bars are $5 \mu \mathrm{m}$.

Fig. 4. Phase behavior of banana-shaped colloidal particles with different curvatures. Confocal microscopy images of colloidal bananas with an average curvature of $\left(\mathrm{A}_{1}-\mathrm{A}_{4}\right) \kappa=$ $0.25 \mu \mathrm{m}^{-1}$ and packing fractions $\phi=0.12,0.26,0.61$ and 0.84 , respectively; $\left(\mathrm{B}_{1}-\mathrm{B}_{4}\right) \kappa=$ $0.10 \mu \mathrm{m}^{-1}$ and $\phi=0.27,0.63,0.79$ and 0.82 , respectively; and $\left(\mathrm{C}_{1}-\mathrm{C}_{4}\right) \kappa=0.07 \mu \mathrm{m}^{-1}$ and $\phi=0.29,0.60,0.67$ and 0.79 , respectively. The top-right overlays on the images show the bananas coloured according to their polar orientation as defined in panel $\mathrm{A}_{5}$ and the legend in panel $A_{1}$. Panels $A_{5}-C_{5}$ show the polar angle distributions of the corresponding confocal microscopy images. Scale bars are $10 \mu \mathrm{m}$.

Fig. 5. Observation of the splay-bend nematic phase. $\left(A_{1}\right)$ Center of mass positions of all the bananas. $\left(A_{2}\right)$ Bananas colored according to the particle orientation as indicated by the white arrows. $\left(A_{3}\right)$ The density profile $\rho(y)$ (blue line) and nematic director field $\hat{\mathbf{n}}(y)$ (white arrows) along the nematic director, $\hat{\mathbf{n}}$, of the system shown in Fig. $4 \mathrm{C}_{3}$. Note that the $y$-axis is parallel to $\hat{\mathbf{n}}$. (B) Confocal microscopy images of the colloidal bananas with curvature $\kappa=0.07 \mu \mathrm{m}^{-1}$ for packing fractions $\phi=0.6,0.64,0.67,0.70$ and 0.79 with $\rho(y)$ and $\hat{\mathbf{n}}(y)$ overlaid. (C) Modulation of the $x$ - and $y$-components of the measured nematic director field along $\hat{\mathbf{n}}$. The solids lines are fits according to the theoretical expression for $\hat{\mathbf{n}}(y)$ (see main text). (D) Pitch-length (in units of the particle length $L$ ) and amplitude $\theta_{0}$ as a function of the packing fraction. (E) Global nematic (NOP) and smectic (SmOP) parameters as a function of the packing fraction. (F) 3D confocal microscopy image $\left(70 \times 70 \times 15 \mu \mathrm{m}^{3}\right)$ of the splay-bend nematic phase. Scale bars are $10 \mu \mathrm{m}$. 